\title{
Communications
}

\section{Rappel de lots}

Coliquifilm $^{\circledast}$, Pommade ophtalmique 3,5 g

\begin{tabular}{ll}
$\mathrm{N}^{\circ}$ d'autorisation: & 46440 \\
\hline Pharmacode: & 1135624 \\
\hline Substance active: & $\begin{array}{l}425 \mathrm{mg} \text { Paraffinum liquidum } \\
\text { et } 573 \mathrm{mg} \text { Vaselinum album } \\
\text { pro } 1 \mathrm{~g}\end{array}$ \\
\hline Lot rappelé: & 86728 \\
\hline
\end{tabular}

Par mesure de précaution, Allergan AG rappelle le lot susmentionné du produit Coliquifilm $^{\circledR}$ en Suisse jusqu'au niveau du commerce de détail. Ce rappel s'effectue en accord avec Swissmedic. Pendant la fabrication des tubes d'aluminium de Coliquifilm, pommade ophtalmique 3,5 g, des particules noires se sont détachées de la matière plastique du capuchon dans de rares cas. Ces particules peuvent éventuellement pénétrer dans la pommade pendant l'administration du produit. Si des particules entrent dans l'œil, les effets indésirables suivants peuvent se produire: douleurs de l'œil, gonflement de l'œil, irritations de l'œil, troubles oculaires, lésions de la cornée.

\section{Produits retournés}

Les clients directs d'Allergan sont priés de retourner les emballages concernés à l'adresse mentionnée ci-dessous:

Kühne + Nagel AG, Warehouse Allergan AG, Contract Logistics, Im Wannenboden 8-10, CH-4133 Pratteln

Les clients qui ne commandent pas directement chez Allergan sont priés de retourner les emballages concernés à leur fournisseur le plus vite possible.

Information: En cas de questions, veuillez nous contacter par tél. au 0442042300 ou par mail à CH_Medinfo[at]allergan.com

\section{Annonces d'effets indésirables}

Pour les annonces d'effets indésirables (EI), Swissmedic recommande d'utiliser le portail prévu à cet effet. Avec le Système électronique Vigilance (ElViS), les EI peuvent être enregistrés directement ou en téléchargeant un fichier xml. Cependant, il est toujours possible d'envoyer les formulaires d'annonce au centre de pharmacovigilance. Ce formulaire se trouve sur le site web de Swissmedic ou peut être commandé directement auprès de Swissmedic (tél. 05846202 23). Toutes les informations requises se trouvent sur www. swissmedic.ch $\rightarrow$ Surveillance du marché $\rightarrow$ Pharmacovigilance.

Nous nous excusons pour les désagréments causés par ce rappel et vous remercions de votre aide et de votre compréhension.

Allergan AG, Zurich

\section{Rappel de lots}

Rappel à titre préventif de tous les lots du produit: Mydocalm ${ }^{\circledR}$ Dragées $150 \mathrm{mg}$

Mesdames, Messieurs,

Nous souhaitons vous informer, qu'en accord avec l'Institut Suisse des Produits Thérapeutiques (Swissmedic), nous effectuons un rappel préventif de tous les lots du produit Mydocalm ${ }^{\circledR}$ Dragées 150 mg jusqu'au niveau du commerce de détail.

Si vous êtes en possession d'emballages de Mydocalm dragées, nous vous prions de les séparer immédiatement de votre stock et de les renvoyer par le biais du circuit de commercialisation (c.-à-d. à votre fournisseur).

Vous recevrez une note de crédit pour la marchandise renvoyée.

Le produit Mydocalm ${ }^{\circledast}$ comprimés pelliculés 150 mg n'est pas concerné par le rappel.

\section{Motif du rappel de lot}

Lors des analyses annuelles de stabilité de certains lots de Mydocalm ${ }^{\circledast}$ Dragées 150 mg, les résultats concernant la dissolution étaient hors des spécifications. En conséquence, et par mesure de précaution, nous rappelons tous les lots de Mydocalm ${ }^{\circledR}$ Dragées 150 mg.

Nous précisons que le produit Mydocalm ${ }^{\circledR}$ Dragées 150 mg, objet de ce retrait, n'est plus distribué en Suisse, depuis septembre 2014. Il a été remplacé par la nouvelle formule galénique du Mydocalm ${ }^{\circledR}$ comprimés pelliculés $150 \mathrm{mg}$.

\section{Annonces d'effets indésirables suspectés d'un médicament}

Pour le signalement de tout effet indésirable (EI), Swissmedic encourage les personnes concernées à utiliser l'outil de déclaration d'effets indésirables développé à cet effet. Avec l'Electronic Vigilance System (EIViS), les EI peuvent être enregistrés directement ou en téléchargeant un fichier xml. Cependant, il est toujours possible d'envoyer les formulaires de signalement au centre de pharmacovigilance. Ce formulaire se trouve sur le site web de Swissmedic ou peut être commandé auprès de Swissmedic (tél. 05846202 23). (Toutes les informations requises se trouvent sur www.swissmedic.ch $\rightarrow$ Surveillance du marché $\rightarrow$ Pharmacovigilance).

Au cas où vous avez des questions, nous restons à votre disposition au numéro de téléphone 0227859500 ou à l'adresse e-mail: info[at]labatec.ch

Nous nous excusons pour les désagréments occasionnés par ce retrait et vous remercions d'ores et déjà de votre soutien et confiance pour toute collaboration future.

Recevez, Mesdames, Messieurs, nos meilleures salutations.

Labatec Pharma SA, Meyrin

\section{Collège de Médecine de Premier Recours CMPR}

\section{Prix CMPR de recherche en médecine de premier recours 2016*}

Spécialité : médecine de premier recours

Critères des travaux soumis: Travaux scientifiques achevés en Suisse ou par des auteurs suisses habitant à l'étranger, qui apportent une contribution importante aux soins de base offerts par le médecin de premier recours, notamment:

- à la qualité du traitement et de la prise en charge des patients de premier recours

- à la pratique médicale des médecins de premier recours (thématique valable, pertinente et réalisable en pratique)

- à la recherche sur le cabinet du médecin de premier recours: fondements, conditions de travail, gestion et préservation des ressources

\section{Prix: $30000 \mathrm{CHF}$}

Le prix sera accordé à un seul lauréat pour son travail scientifique ou alors il sera réparti sur plusieurs auteurs (sans possibilité de recours devant les tribunaux).

Participant(e)s: Auteurs en Suisse ou personnes suisses habitant à l'étranger ayant achevé un travail scientifique majeur sur la 
médecine de premier recours dans les trois dernières années.

\section{Délai de soumission: 1 décembre 2015}

Remise du prix: Lors des colloques de formation continue du CMPR, les 23 et 24 juin 2016 à Lucerne et le 8 septembre 2016 à Lausanne.

Comité: Jury indépendant, nommé par le conseil de fondation du CMPR

Information: Détail des conditions de participation: dossier à fournir sous forme électronique ou de $\mathrm{CD}$ (pas de disquettes) et sous forme papier: formulaire d'inscription (www.kollegium.ch/rd/f.html), curriculum vitae de l'auteur principal, manuscrit, brève lettre d'accompagnement «importance de ce travail pour la médecine de premier recours».

Nous ne prendrons pas en considération les travaux ayant déjà été soumis une fois et il n'y aura pas d'échange de courrier au sujet de l'attribution des prix.
Lieu de contact: Collège de médecine de premier recours CMPR, Secrétariat de la Recherche en médecine de premier recours, Rue de l'Hôpital 15, CP 1552, 1701 Fribourg, khm[at]hin.ch; www.kollegium.ch/rd/f.html

* offert par la maison Mepha Pharma SA

Société Suisse de Pharmacologie et Toxicologie Cliniques (SSPTC)

\section{Prix de la SSPTC}

Dans le but de supporter et promouvoir la recherche de jeunes scientifiques dans le domaine de la pharmacologie et toxicologie cliniques, la Société Suisse de Pharmacologie et Toxicologie Cliniques (SSPTC) attribue chaque année un prix de 1000 francs pour la meilleure thèse dans ce domaine. Des thèmes possibles sont des travaux de thèse (MD, PhD, master) théoriques, expérimentaux ou cliniques dans le domaine de la pharmacologie et toxicologie clinique. La thèse doit avoir été acceptée par une université suisse durant les deux années précédant l’année de la soumission. L'auteur(e) ne devrait pas être plus âgé(e) que 40 ans. L'appartenance à la SSPTC n'est pas une condition pour l'attribution de ce prix.

Le manuscrit de la thèse (si supérieur à 10 pages $\mathrm{A} 4$ joindre un résumé) dans une des quatre langues nationales ou en anglais doit être envoyé au secrétaire de la SSPTC (adresse voir www.clinpharm.ch). Une copie de l'imprimatur, curriculum vitae ainsi qu'une liste éventuelle des publications doivent être également joints au dossier. L’envoi doit être fait sous forme électronique (fichier pdf).

Le délai de soumission est le 15.2.2016.

Swiss Quality Award

INNOVATIONS IN HEALTHCARE

\title{
ENVOYEZ VOS PROJETS AVANT LE 8 JANVIER 2016
}

\author{
Le Swiss Quality Award récompense les meilleures innovations en matière \\ de qualité dans le système de santé. Participez et enregistrez votre projet sur \\ le site www.swissqualityaward.ch.
}

